\title{
A Critical Assessment of Multiple Scattering Expansions*
}

\author{
D. Sébilleau ${ }^{\dagger}$ \\ Groupe Théorie, Département Matériaux-Nanosciences, \\ Institut de Physique de Rennes, UMR CNRS-UR1 6251, \\ Université de Rennes-1, 35042 Rennes cedex, France \\ K. Hatada \\ CNISM, Sezione di Fisica, Scuola di Scienze e Tecnologie, \\ Università di Camerino, I-62032 Camerino (MC), Italy and \\ Theory Group, INFN-LNF, c. p. 13, I-00044 Frascati, Italy \\ H. -F. Zhao \\ Beijing Synchrotron Radiation Facility, Institute of High Energy Physics, \\ Chinese Academy of Sciences, PO Box 918, Beijing 100049, People's Republic of China \\ C. R. Natoli \\ Theory Group, INFN-LNF, c. p. 13, I-00044 Frascati, Italy \\ (Received 3 September 2012; Accepted 17 October 2012; Published 3 November 2012)
}

\begin{abstract}
We propose a comparative and critical assessment of multiple scattering expansions. The so-called multiple scattering series expansion is much used in the description of spectroscopies at higher energies. However, it is plagued with convergence problems when operated at lower energies. We compare this method to related methods that can be found in the literature, relying both on finite and infinite expansions. After discussing the pros and cons of these methods, we establish a simple alternative to multiple scattering series expansion which has a wider and faster range of convergence.
\end{abstract}

[DOI: 10.1380/ejssnt.2012.599]

Keywords: Green's function methods; Scattering theory; Convergence problems

\section{INTRODUCTION}

Convergence of series expansions is an old problem in mathematics and physics. Many approaches have been proposed over the centuries to control or improve it. In multiple scattering theory, which is used in many areas of physics to describe the interaction of a wave with a media composed of distinguishable objects, such series expansions are currently used as one of the main algorithms to solve the problem.

In this article, we propose to explore the realm of multiple scattering expansions in order to identify better candidates than the currently used method to optimize as much as possible the computing time, and to reach convergence when the latter method diverges. The so-called multiple scattering series expansion method, which many codes computing the cross-section at intermediate kinetic energies of spectroscopies involving electrons are based on, does not converge all the time. Moreover, when the size of the cluster used to represent the material increases, calculations can become very cumbersome. Material characterization nowadays tends more and more to the nanoscopic scale, which implies extremely large numbers of atoms to correctly represent the material. This pushes the limits of the multiple scattering series expansion outside its do-

\footnotetext{
*This paper was presented at the conference "XAFS theory and nano particles", Chiba University, Chiba, Japan, July 18-20, 2012. ${ }^{\dagger}$ Corresponding author: didier.sebilleau@univ-rennes1.fr
}

main of feasibility. To overcome this problem, we will consider in this article some possible candidates for the replacement of the standard series expansion.

Although we will derive here a new type of series expansion which we call renormalized multiple scattering series expansion, we consider the present article more as an invitation to explore new pathways in the computation of multiple scattering expansions rather than as the presentation of new results. This is what we call in the title "a critical assessment".

In the next section, we will give a brief outline of multiple scattering theory within the scattering path operator framework. Section III will be devoted to the study of the convergence properties of the multiple scattering series expansion in order to point out its failures. Alternative expansions that seem promising will be described in Sec. IV. Section V will propose a new and simple approach to the problem. A critical comparison of these different expansions will be given in Sec. VI before our conclusions.

\section{THE SCATTERING PATH OPERATOR MULTIPLE SCATTERING FRAMEWORK}

Multiple scattering is a very powerful tool used in many fields of science such as nuclear/particle physics, acoustics, medical physics, atomic physics, geophysics, etc. It can be applied each time a physical process can be described by a wave equation. Scattering theory is based on the description of the interaction of a wave with a collec- 
tion of obstacles, which can be transformed into a Green's function equation. If we call $G$ the total Green operator associated to the total wave equation (including the obstacles), and which represents the solution of the system, this operator can then be expressed solely in terms of two key ingredients:

- the propagator $G_{o}$ of this wave in the absence of obstacles (free propagation). This propagator, also called resolvent or Green's operator, is purely a characteristic of the wave equation.

- the transition operator $T$ which describes the effect of the obstacle on the incident wave. These operators are characteristics of the obstacles.

The case the present authors are interested in is the description of the interaction of an electron with a collection of atoms. This is needed to model spectroscopies such as photo-emission, X-ray absorption or electron diffraction within multiple scattering theory [1]. The so-called KKR theory of electronic structure [2] relies also on the same model, although it deals with bound states rather than extended states. The corresponding equation is the Schrödinger equation. In the time-independent (steadystate) case, the free electron Schrödinger equation reduces to the well-known Helmholtz equation

$$
\vec{\nabla}^{2} \psi+k^{2} \psi=0
$$

This equation, together with its perturbed version (presence of obstacles in the system), is the one on which our multiple scattering theory is based. The consequence is that any physical process described by the same Helmholtz equation (such as the acoustic wave equation, or the heat equation) can be formulated within the same formalism as the one we will outline now, with the only difference of the response of the obstacles to the incoming wave ( $T$ matrices).

Our aim in this section is not to re-derive all multiple scattering theory, but simply to explain how series expansions can occur within this framework. Therefore, for a proper derivation of the basic equations, and the relation to the Schrödinger equation, we refer the reader to a review centered on spectroscopies [1]. However, prior to proceeding, we need to recall a few basic definitions of scattering theory. A general presentation of multiple scattering theory in the operator representation has been proposed in [3] from which we take the main definitions given here.

Starting from the Schrödinger equation with Hamiltonian $H=H_{o}+V$, we can write the propagator $G$ as

$$
G(z)=(z-H)^{-1},
$$

where $z$ is any complex number. In multiple scattering theory, $z$ is taken as the square of the wave number $k$. It is identical to the mathematical quantity called resolvent of $H$ and noted $R_{H}(z)$.

It can be deduced from the free electron propagator $G_{O}$ associated to $H_{o}$ by the so-called Dyson equation

$$
G=G_{o}+G_{o} V G=G_{o}+G V G_{o} .
$$

The transition operator $T$ is then defined by

$$
T=V+V G V
$$

so that $G_{o} T=G V$ and the Dyson equation (3) transforms as $G=G_{o}+G_{o} T G_{o}$.

In the multiple scattering case, we write $V$ as a superposition of atomic potentials: $V=\sum_{\mathrm{i}} V_{\mathrm{i}}$. Note however that $T \neq \sum_{\mathrm{i}} T_{\mathrm{i}}$. We denote $G_{\mathrm{i}}$ the propagator in the presence of potential $V_{\mathrm{i}}$ alone. From Dyson equation and Eq. (2), we deduce that it writes as

$$
G_{\mathrm{i}}=G_{o}+G_{o} T_{\mathrm{i}} G_{o}=\left(k^{2}-H_{o}-V_{\mathrm{i}}\right)^{-1}
$$

and likewise for the atomic transition operator

$$
T_{\mathrm{i}}=V_{\mathrm{i}}+V_{\mathrm{i}} G_{\mathrm{i}} V_{\mathrm{i}}
$$

which represents scattering by atom i alone.

With the equipment of $\left(G_{O}, T\right)$ to describe the physical process, it has been then demonstrated that the scattering path operator defined as

$$
\boldsymbol{\tau}=\left(\boldsymbol{T}^{-1}-\boldsymbol{G}_{\boldsymbol{o}}\right)^{-1}
$$

is the central quantity to access the cross-section of many spectroscopies. Actually, it is shown in Ref. [1] that any cross section of a spectroscopy involving an electron as the probe in a material can be expressed in terms of specific matrix elements of $\boldsymbol{\tau}$. Here, bold quantities represent matrices. Generally, the basis used to make the calculation is the spherical wave basis. This is the one we will use in the following in our calculations and therefore, matrices should be understood as composed of the matrix elements of the corresponding operator in this basis.

This scattering path operator was introduced in condensed matter theory by Gyorffy and Stott [4], but can be traced back to Faddeev [5] in nuclear physics.

Equation (7) results from the factorization of the equation of motion

$$
\bar{\tau}^{\mathrm{ji}}=\bar{T}_{\mathrm{i}} \delta_{\mathrm{ji}}+\sum_{\mathrm{k} \neq \mathrm{j}} \bar{T}_{\mathrm{j}} G_{o} \bar{\tau}^{\mathrm{ki}},
$$

which itself can be obtained from the definition of the scattering path operator

$$
\bar{\tau}^{\mathrm{ji}}=\bar{V}_{\mathrm{i}} \delta_{\mathrm{ji}}+\bar{V}_{\mathrm{j}} G \bar{V}_{\mathrm{i}}
$$

This definition is extremely rewarding as it gives a direct physical interpretation of the scattering path operator element $\tau^{\mathrm{ji}}$ : it describes all the possibilities an electron has to go from atom $i$ to atom $\mathrm{j}$. Hence the name of this operator. Here, the overbar is simply a reminder that, because atom $\mathrm{i}$ and atom $\mathrm{j}$ do not necessarily coincide with the arbitrary origin we choose, the corresponding operator contains translation operators referring to this origin. We note also that

$$
T=\sum_{\mathrm{i}, \mathrm{j}} \bar{\tau}^{\mathrm{ij}}
$$

Equation (7) can be rewritten as

$$
\tau=\boldsymbol{T}\left(\boldsymbol{I}-G_{o} T\right)^{-1}
$$

where $\boldsymbol{I}$ is the identity matrix.

This equation leads directly to two possible algorithms to compute the matrix elements of the scattering path operator $\tau$ : 
- matrix inversion: the matrices are filled up and matrix $\boldsymbol{\tau}$ is obtained by numerical inversion of the socalled multiple scattering matrix $\left(\boldsymbol{I}-\boldsymbol{G}_{\boldsymbol{o}} \boldsymbol{T}\right)$. This method is exact from a purely scattering point of view, but its use can be restricted due to the size of the matrix that has to be stored. The size $N$ of the problem is typically equal to the number of atoms $N_{a t}$ in the cluster used for the calculations times the number of basis functions used to expand the scattering wave function on each atomic site which amounts to $\left(l_{\max }+1\right)^{2}$, where $l_{\max }$ is the angular momentum cut-off on each atom. In this case, storage will scale like $N^{2}$ and numerical inversion like $N^{3}$, which can soon become intractable.

- multiple scattering series expansion. It is obtained by performing a Taylor-like expansion on the matrix inverse, leading to

$$
\boldsymbol{\tau}=\boldsymbol{T}\left(\boldsymbol{I}+\boldsymbol{G}_{\boldsymbol{o}} \boldsymbol{T}+\left(\boldsymbol{G}_{\boldsymbol{o}} \boldsymbol{T}\right)^{2}+\left(\boldsymbol{G}_{\boldsymbol{o}} \boldsymbol{T}\right)^{3}+\cdots\right) .
$$

This approach, generally called the Watson expansion, has been first introduced in multiple scattering nuclear physics by Watson [6] to compute $T$ in terms of the individual nuclear $T_{\mathrm{i}}$. It is only valid when the expansion converges.

Note that the approach taken here implicitily considers the case of spherical potentials. But multiple scattering theory can be generalized to the case of non overlapping potential of any shape (including non-local potentials) and multichannel potentials (to account for electron correlation) [7].

\section{THE CONVERGENCE OF WATSON MULTIPLE SCATTERING EXPANSION}

Following a suggestion by Natoli and Benfatto [8], two of us implemented in a recent paper [9] a way to measure and check the convergence of the Watson multiple scattering expansion. As $\boldsymbol{G}_{\boldsymbol{o}} \boldsymbol{T}$ in Eq. (12) is diagonalizable, we can write it as $\boldsymbol{G}_{\boldsymbol{o}} \boldsymbol{T}=\boldsymbol{S} \boldsymbol{D} \boldsymbol{S}^{-1}$, where $\boldsymbol{D}$ is a diagonal matrix, so that expansion Eq. (12) becomes

$$
\left(\boldsymbol{I}-\boldsymbol{G}_{\boldsymbol{o}} \boldsymbol{T}\right)^{-1}=\boldsymbol{S}\left(\boldsymbol{I}+\boldsymbol{D}+\boldsymbol{D}^{2}+\boldsymbol{D}^{3}+\cdots\right) \boldsymbol{S}^{-1} .
$$

The expansion in terms of the diagonal matrix $\boldsymbol{D}$ converges if, for all eigenvalues $\lambda_{i}$ of $\boldsymbol{D}$ the expansion

$$
\left(1-\lambda_{i}\right)^{-1}=1+\lambda_{i}+\lambda_{i}^{2}+\lambda_{i}^{3}+\cdots
$$

is convergent.

Defining the spectral radius $\rho\left(\boldsymbol{G}_{\boldsymbol{o}} \boldsymbol{T}\right)$, i.e. the radius of the spectrum of $\boldsymbol{G}_{\boldsymbol{O}} \boldsymbol{T}$ as

$$
\rho\left(\boldsymbol{G}_{\boldsymbol{o}} \boldsymbol{T}\right)=\max _{i}\left|\lambda_{i}\right| .
$$

We deduce from (14) that the condition for the convergence of Watson multiple scattering expansion is

$$
\rho\left(\boldsymbol{G}_{\boldsymbol{o}} \boldsymbol{T}\right)<1 .
$$

Moreover, we deduce from Eq. (12) that the lower $\rho\left(\boldsymbol{G}_{\boldsymbol{o}} \boldsymbol{T}\right)$, the faster the convergence.
This tool was applied in [9] to check the convergence of the Watson expansion in two different systems, $\mathrm{Cu}(111)$ and $\mathrm{TiO}_{2}(110)$. The results are shown in Fig. 1 together with the comparison of photoelectron diffraction (PED) calculations at selected energies $(25 \mathrm{eV}$ and $45 \mathrm{eV})$ using single scattering approximation on the left hand side (first order of Watson expansion) and numerical matrix inversion (Eq. (11)) on the right hand side. We see that the spectral radii of $\mathrm{Cu}(111)$ and $\mathrm{TiO}_{2}(110)$ are quite different. Furthermore, their respective values at the selected energies explain why single scattering and full multiple scattering (matrix inversion) agree for $\mathrm{Cu}(111)$ while they strongly differ in the case of $\mathrm{TiO}_{2}(110)$ (in this latter case the scattering expansion even diverges at $25 \mathrm{eV}$ ).

The most striking feature in this calculation is that the spectral radius does not decrease in a monotonous way as we would have expected, but oscillates. Another interesting feature is that in the first $120 \mathrm{eV}$, the spectral radii of $\mathrm{Cu}(111)$ and $\mathrm{TiO}_{2}(110)$ are completely different while at higher energies, they are basically identical.

These oscillations result from an interplay of the chemical/electronic structure contained in $\boldsymbol{T}$ and the crystallographic structure contained in $\boldsymbol{G}_{\boldsymbol{o}}$. At higher energies, the behaviour of the spectral radius is dominated by the atomic number $Z$ although the influence of the geometric structure cannot be discarded as we found out in preliminary studies. At lower energies (below $100 \mathrm{eV}$ ) the analysis is still more complex, but we found that even for the same geometric structure and outer shell structure, variations in the lattice parameter were responsible for dramatic changes in the oscillations.

Another parameter that strongly affects the oscillations of the spectral radius is the size of the cluster. This does not come as a surprise as $\rho\left(\boldsymbol{G}_{\boldsymbol{o}} \boldsymbol{T}\right)$ can be seen as a measure of the multiple scattering within the system. As such, we expect it to increase with the size of the cluster. This is actually what happens as can be seen from Fig. 2 .

Interestingly enough, above a certain cluster size where all the features of the oscillations are present (51 atoms in our case), the evolution of the spectral radius essentially consists in a vertical rigid shift of the 51 atom curve. It seems moreover that this shift is proportional to the radius of the cluster. More tests are necessary to assess this behavior, but if verified, it would give a straightforward way to get an estimate of the spectral radius at a given energy: perform the calculation for a reasonably sized cluster and then scale it by the ratio of the cluster sizes.

This demonstrates that one has to be very cautious when using the Watson series expansion. Indeed, when increasing the size of the cluster, even with a rather low number of atoms such as 153, divergence can occur at higher energies.

\section{MULTIPLE SCATTERING EXPANSIONS}

We review now some of the common alternative multiple scattering expansions that can be found in the literature. We do not pretend to be exhaustive in this review, but instead we focus on some of them that seems of interest to us, and we will discuss why in Sec. VI. To simplify the notation, we will write now the kernel matrix $\boldsymbol{G}_{\boldsymbol{o}} \boldsymbol{T}$ as $\boldsymbol{K}$. 


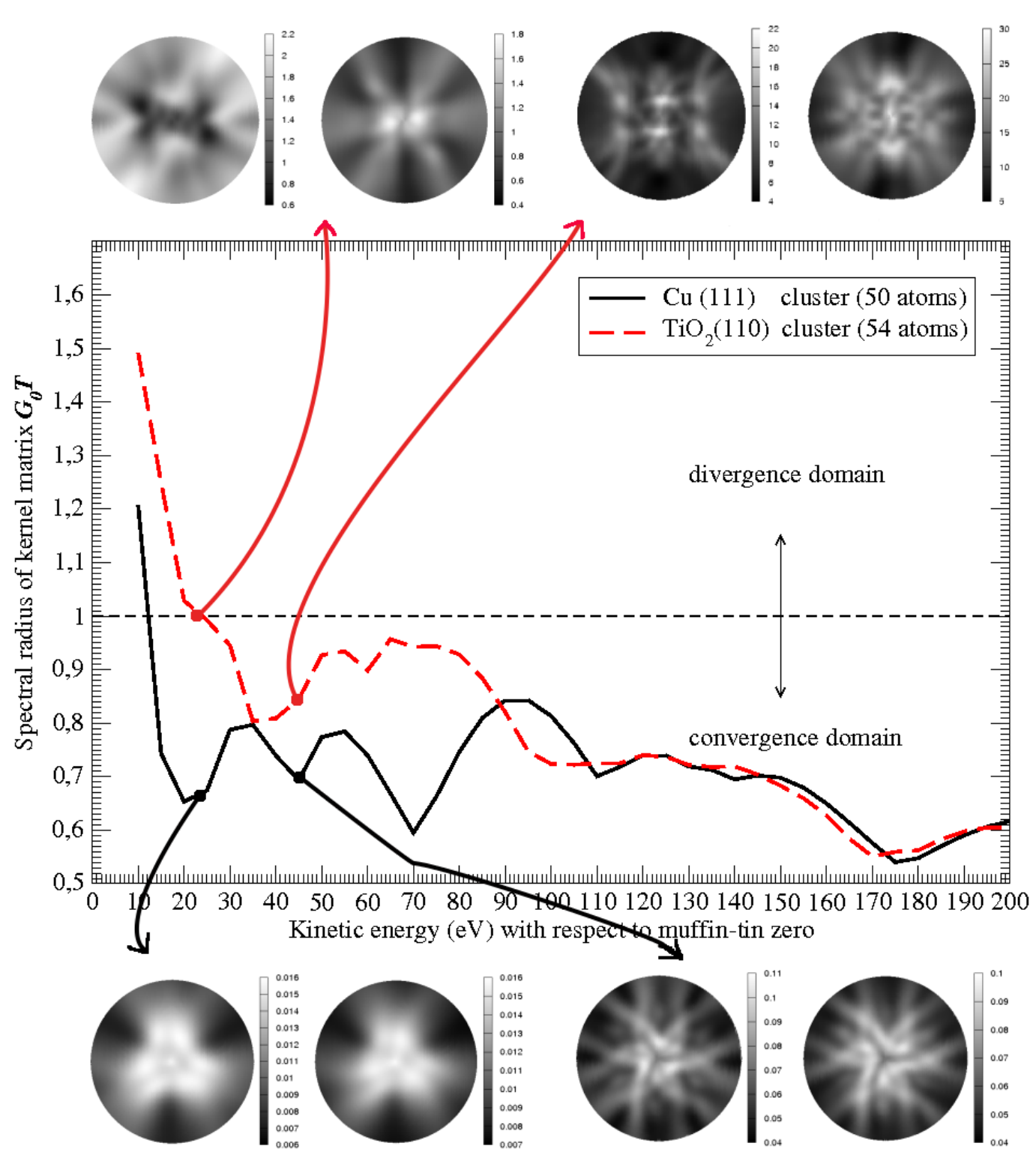

FIG. 1: Evolution of the spectral radius for $\mathrm{Cu}(111)$ and $\mathrm{TiO}_{2}(110)$. PED calculations are also presented at $25 \mathrm{eV}$ and $45 \mathrm{eV}$. Left hand side calculations correspond to single scattering while right hand side ones are computed using full inversion of the multiple scattering matrix.

The important point is that all multiple scattering expansions need not be infinite like Watson's. The latter is based on perturbation theory, and this is the fundamental reason why convergence can be a problem as we discussed it in the previous section.

\section{A. Alternative infinite expansions}

In a famous paper written in 1962, Löwdin explored alternative ways to compute the inverse of the matrix $(\boldsymbol{I}-\boldsymbol{K})^{-1}$. We mention two of his approaches now.

\section{Neumann expansion}

Löwdin introduces Neumann expansion as

$$
(\boldsymbol{I}-\boldsymbol{K})^{-1}=\sum_{k=0}^{+\infty}(\boldsymbol{I}-\boldsymbol{N}+\boldsymbol{N} \boldsymbol{K})^{k} \boldsymbol{N}
$$

This result is valid for any matrix $\boldsymbol{N}$. Its demonstration is easy. However, he did not give any reference to it and therefore we will keep calling it Neumann expansion.

The interest of this expansion is that it is expressed in terms of the powers of the alternative matrix $(\boldsymbol{I}-\boldsymbol{N}+$ $\boldsymbol{N} \boldsymbol{K})$. This opens the way to the quest of $\boldsymbol{N}$ for which $\rho(\boldsymbol{I}-\boldsymbol{N}+\boldsymbol{N} \boldsymbol{K})<\rho(\boldsymbol{K})$ in which case the Neumann 


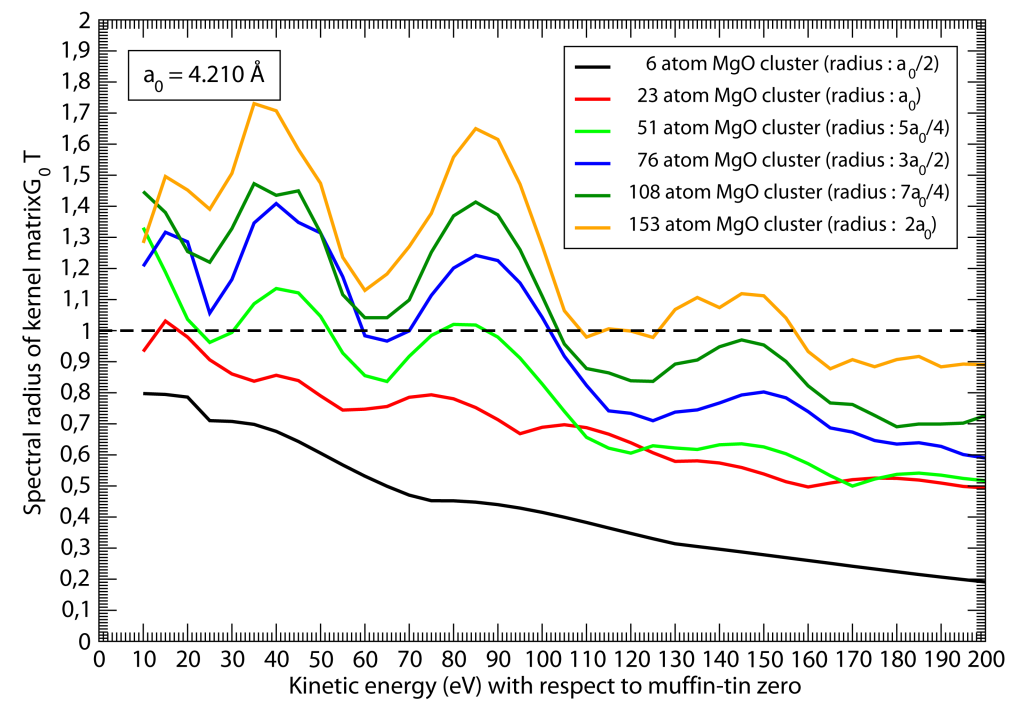

FIG. 2: Evolution of the spectral radius as a function of the cluster radius.

expansion would converge faster that the Watson expansion.

It is then straightforward to demonstrate that

$$
(\boldsymbol{I}-\boldsymbol{K})^{-1}=(\boldsymbol{I}-\boldsymbol{M})^{-1} \boldsymbol{N}=\boldsymbol{N}^{\prime}\left(\boldsymbol{I}-\boldsymbol{M}^{\prime}\right)^{-1}
$$

with $\boldsymbol{M}=\boldsymbol{I}-\boldsymbol{N}(\boldsymbol{I}-\boldsymbol{K})$ and $\boldsymbol{M}^{\prime}=\boldsymbol{I}-(\boldsymbol{I}-\boldsymbol{K}) \boldsymbol{N}^{\prime}$. Here again $\boldsymbol{N}$ and $\boldsymbol{N}^{\prime}$ represent any matrix. We will make use of this form in Sec. $\mathrm{V}$ to classify the new expansions we will derive.

This type of expansion has been studied in detail by Tanabe [11] and others [12] in the context of generalized inverses.

\section{Second order iteration}

In his famous paper [10], Löwdin proposed an alternative scheme to compute $(\boldsymbol{I}-\boldsymbol{K})^{-1}$ using a series expansion. He called it second order iteration expansion. Let us note

$$
\boldsymbol{S}_{n}=\sum_{k=0}^{n} \boldsymbol{K}^{k}
$$

The partial sum at order $n$ of the Taylor expansion of $(\boldsymbol{I}-\boldsymbol{K})^{-1}$. Löwdin introduced the companion matrix $\boldsymbol{S}_{n}^{*}$ defined by

$$
\boldsymbol{S}_{n}^{*}=2 \boldsymbol{S}_{n}-\boldsymbol{S}_{n}(\boldsymbol{I}-\boldsymbol{K}) \boldsymbol{S}_{n} .
$$

It is then straightforward to show that

$$
\boldsymbol{S}_{n}^{*}=\boldsymbol{S}_{2 n+1},
$$

which means that $\boldsymbol{S}_{n}^{*}$ is the partial sum at order $(2 n+1)$ of the same expansion. The second order iteration matrix $\boldsymbol{S}_{n}^{*}$ satisfies the following recurrence relations

$$
\left\{\begin{aligned}
\boldsymbol{S}_{n+1}^{*} & =\boldsymbol{S}_{n}^{*}\left(\boldsymbol{I}+\boldsymbol{K}^{2}\right)-\boldsymbol{S}_{n-1}^{*} \boldsymbol{K}^{2} \\
& =\boldsymbol{S}_{n}^{*}+\left(\boldsymbol{S}_{n}^{*}-\boldsymbol{S}_{n-1}^{*}\right) \boldsymbol{K}^{2}
\end{aligned}\right.
$$

with the initial values

$$
\left\{\begin{array}{l}
\boldsymbol{S}_{-1}^{*}=\mathbf{0} \\
\boldsymbol{S}_{0}^{*}=\boldsymbol{S}_{1} \\
\boldsymbol{S}_{1}^{*}=\boldsymbol{S}_{0}^{*}\left(\boldsymbol{I}+\boldsymbol{K}^{2}\right)
\end{array}\right.
$$

\section{B. Alternative finite expansions}

Multiple scattering finite expansions have the advantage that they always converge as we can always sum up to the last term: for a given operator $A$, they can be written as

$$
A=\sum_{n=1}^{N} A^{(n)}
$$

were $N$ is a fixed number (number of atoms, size of matrix, ...). We consider now some of them which we will further discuss in Section VI. For application, we will be interested in $A=T$ or $\boldsymbol{A}=\boldsymbol{\tau}$.

\section{Correlation expansion}

Correlation expansion was proposed for the first time in the multiple scattering theory of nuclear physics by Thaler and his coworkers $[13,14]$. It is based on a combinatorial identity that is demonstrated in appendix B of [14]. It is a very general theorem that relies purely on mathematical grounds and not on perturbation theory as the Watson expansion. Let $A$ be a quantity that depends solely on a set of parameters $\{\mathrm{i}, \mathrm{j}, \mathrm{k}, \cdots, \mathrm{N}\} . A$ and the parameters can be anything. Then according to the correlation expansion theorem, we can write 


$$
\begin{aligned}
A= & \sum_{\mathrm{i}=1}^{N} A^{\mathrm{i}}+\sum_{\mathrm{i}<\mathrm{j}}^{N}\left[A^{\mathrm{ij}}-A^{\mathrm{i}}-A^{\mathrm{j}}\right]+\sum_{\mathrm{i}<\mathrm{j}<\mathrm{k}}^{N}\left[A^{\mathrm{ijk}}-A^{\mathrm{ij}}-A^{\mathrm{ik}}-A^{\mathrm{jk}}+A^{\mathrm{i}}+A^{\mathrm{j}}+A^{\mathrm{k}}\right]+ \\
& \sum_{\substack{\mathrm{i}<\mathrm{j}<\mathrm{k}<\mathrm{l}\\
}}\left[A^{\mathrm{ijkl}}-A^{\mathrm{ijk}}-A^{\mathrm{ijl}}-A^{\mathrm{ikl}}-A^{\mathrm{jkl}}+A^{\mathrm{ij}}+A^{\mathrm{ik}}+A^{\mathrm{il}}+A^{\mathrm{jk}}+A^{\mathrm{jl}}+A^{\mathrm{kl}}-A^{\mathrm{i}}-A^{\mathrm{j}}-A^{\mathrm{k}}-A^{1}\right]+
\end{aligned}
$$

This identity is always valid as long as we can identify $A$ with the last term $A^{\mathrm{ijk} \cdots \mathrm{N}}$. It comes from the fact that at each order above second order, we substract part of the previous order so that in the end, we are only left with the last term $A^{\mathrm{ijk} \cdots \mathrm{N}}$. In Ref. [15], Zhao and coworkers showed how this abstract expansion could be used to compute the scattering path operator $\boldsymbol{\tau}$. We refer the reader to this article for the exact derivation of the scattering path operator correlation expansion recalling however their main results concerning the convergence of this expansion. Figure 3 shows the comparison of the convergence of correlation expansion with Watson series expansion in a test case, the cross section of PED for a cluster of $\mathrm{MgO}(001)$ containing 23 atoms at $184 \mathrm{eV}$. The calculations have been performed with the MsSpec code [20]. These results are compared to the exact calculation obtained from the inversion of the multiple scattering matrix. Both expansions converge, but we see that correlation expansion converges faster at the same order.

Figure 4 corresponds to a cross-section calculated at 21 $\mathrm{eV}$, for the same cluster. Here, it has not been possible to plot the results of Watson single and double scattering calculations as the results blow up completely due to the strong divergence of the multiple scattering expansion, as detailed in Sec. III. For correlation expansion, although we need to go much further in the expansion, we verify that there is convergence, as expected from the SicilianoThaler theorem (25).

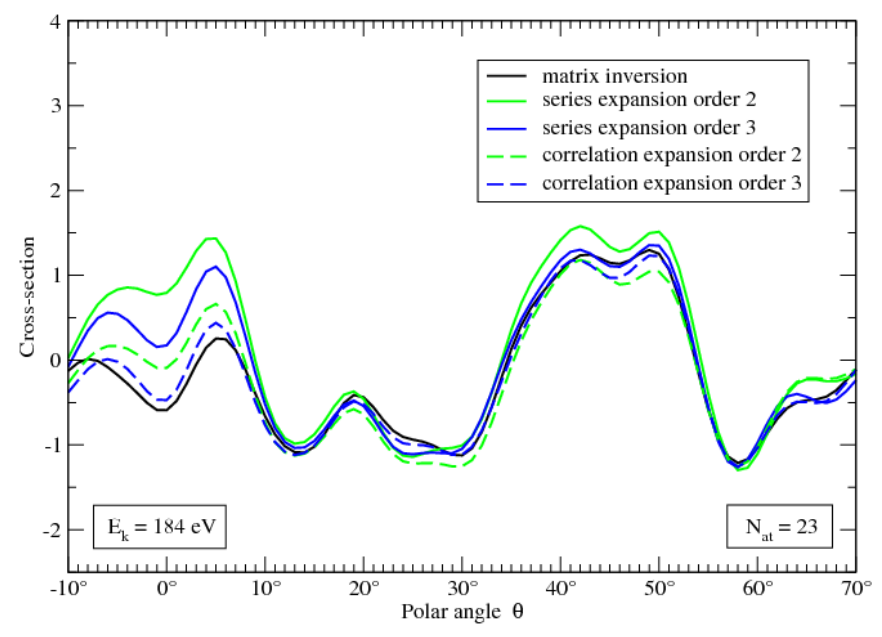

FIG. 3: Comparison of the convergence of correlation expansion $v s$ Watson series expansion for polar photoelectron diffraction on $\mathrm{MgO}(001)$ at $184 \mathrm{eV}$, with the result of matrix inversion given as a reference.
More systematic calculations have been performed in Ref. [15], and the authors conclude that correlation expansion does indeed converge all the time and that in all the cases treated, it provides a faster convergence.

\section{Local-field multiple scattering expansion}

This finite expansion was proposed in 1981 in nuclear physics by Wallace and coworkers [16]. To express it, we need to give a few definitions that will allow to simplify the notation. First, we denote by

$$
V^{\mathrm{ij}}=V-V_{\mathrm{i}}-V_{\mathrm{j}}
$$

the complement to the potential $\left(V_{\mathrm{i}}+V_{\mathrm{j}}\right)$. Then, we introduce the idea of a local field $\phi$ as

$$
G_{\mathrm{i}}[\phi]=\left(z-H_{o}-V_{\mathrm{i}}-\phi\right)^{-1}
$$

Therefore, $G_{\mathrm{i}}[\phi]$ represents the propagator associated to atom $\mathrm{i}$ in the presence of the local field $\phi$. Under this definition, $G_{\mathrm{i}}$ defined by Eq. (5) is equal to $G_{\mathrm{i}}[0]$. This definition generalizes the local propagators $G_{\mathrm{i}}$. We define also the local field transition operator

$$
T_{\mathrm{i}}[\phi]=V_{\mathrm{i}}+V_{\mathrm{i}} G_{\mathrm{i}}[\phi] V_{\mathrm{i}}
$$

Here again, we have $T_{\mathrm{i}}[0]=T_{\mathrm{i}}$. Then, it can be demon-

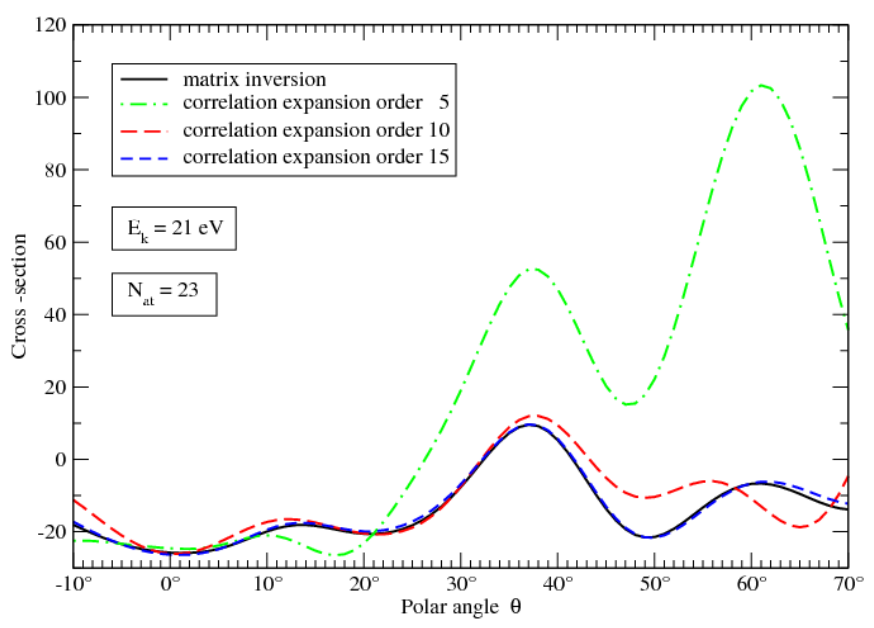

FIG. 4: Convergence of correlation expansion for polar photoelectron diffraction on $\mathrm{MgO}(001)$ at $21 \mathrm{eV}$, with the result of matrix inversion given as a reference. 
strated that

$$
\begin{aligned}
T & =\sum_{\mathrm{i}=1}^{N} T_{\mathrm{i}}\left[V^{\mathrm{i}}\right]+\sum_{\mathrm{i}, \mathrm{j}}^{N} T_{\mathrm{i}}\left[V^{\mathrm{ij}}\right] G_{o} T_{\mathrm{j}}\left[V^{\mathrm{j}}\right] \\
& +\sum_{\mathrm{i}, \mathrm{j}, \mathrm{k}}^{N} T_{\mathrm{i}}\left[V^{\mathrm{ijk}}\right] G_{o} T_{\mathrm{j}}\left[V^{\mathrm{jk}}\right] G_{o} T_{\mathrm{k}}\left[V^{\mathrm{k}}\right]+\cdots
\end{aligned}
$$

This is the local-field multiple scattering expansion. It is a finite expansion with $N$ terms, $N$ being the number of atoms.

\section{Leverrier-Faddeev expansion}

The Leverrier-Faddeev algorithm was originally designed by Leverrier in 1840 to compute the characteristic polynomial of a matrix. It was rediscovered several times later until widely popularized by Faddeev [17].

As given by Hou [18], the resolvent $\boldsymbol{R}_{\boldsymbol{K}}(z)$ defined by $(z \boldsymbol{I}-\boldsymbol{K})^{-1}$, can be written for $z=1$ as

$$
(\boldsymbol{I}-\boldsymbol{K})^{-1}=\frac{1}{p(1)} \sum_{k=1}^{n} \boldsymbol{B}_{k}
$$

where $n$ is the size of matrix $\boldsymbol{K} \cdot p(\lambda)$ is the characteristic polynomial of $\boldsymbol{K}$ given by

$$
p(\lambda)=a_{0} \lambda^{n}+a_{1} \lambda^{n-1}+\cdots+a_{n-1} \lambda+a_{n} .
$$

The Leverrier-Faddeev algorithm provides the way to compute recursively the $\boldsymbol{B}_{k}$ matrices and the coefficients $a_{k}:$

$$
\left\{\begin{aligned}
\tilde{\boldsymbol{B}}_{k} & =\boldsymbol{K} \boldsymbol{B}_{k} \\
a_{k} & =-\frac{1}{k} \operatorname{Tr}\left[\tilde{\boldsymbol{B}}_{k}\right] \quad \text { and } \quad \boldsymbol{B}_{1}=\boldsymbol{I} \\
\boldsymbol{B}_{k+1} & =\tilde{\boldsymbol{B}}_{k}+a_{k} \boldsymbol{I}
\end{aligned}\right.
$$

This algorithm was proposed to compute exactly the inverse, or eigenvalues. However, we see from Eq. (30) that we can consider it as an expansion. In terms of powers of $\boldsymbol{K}$, the expansion can be rewritten as

$$
\begin{aligned}
(\boldsymbol{I}-\boldsymbol{K})^{-1}= & \frac{1}{p(1)}\left[\boldsymbol{I}+\left(a_{1} \boldsymbol{I}+\boldsymbol{K}\right)+\right. \\
& \left.\left(a_{2} \boldsymbol{I}+a_{1} \boldsymbol{K}+\boldsymbol{K}^{2}\right)+\cdots\right] .
\end{aligned}
$$

\section{THE RENORMALIZED MULTIPLE SCATTERING EXPANSIONS}

The new method we introduce now is based on an idea developped by Janiszowski [19] to derive an algorithm for matrix inversion in processors with limited calculation capabilities. Let us suppose that we are able somehow to relate $(\boldsymbol{I}-\boldsymbol{K})^{-1}$ to the inverse $(\boldsymbol{I}-\boldsymbol{G})^{-1}$ involving an auxilliary matrix $\boldsymbol{G}$. If in addition this matrix $\boldsymbol{G}$ satisfies $\rho(\boldsymbol{G})<\rho(\boldsymbol{K})$, then we have an expansion in $\boldsymbol{G}$ that will converge faster, and we can use it to expand $(\boldsymbol{I}-\boldsymbol{K})^{-1}$.

To start with, we introduce the auxilliary matrix $\boldsymbol{G}$ by

$$
\boldsymbol{G}=(1-\omega) \boldsymbol{I}+\omega \boldsymbol{K}
$$

where $\omega$ is a complex number. $\boldsymbol{G}$ can be viewed as a mixing between the kernel matrix $\boldsymbol{K}$ and the identity matrix.

It is then straightforward to demonstrate that

$$
(\boldsymbol{I}-\boldsymbol{K})^{-1}=\omega(\boldsymbol{I}-\boldsymbol{G})^{-1}
$$

The quantity $\omega$ can be viewed as a renormalization parameter. The Taylor series expansion in terms of the $\boldsymbol{G}$ matrix is obviously different from the one in terms of the kernel matrix $\boldsymbol{K}$, except for the value $\omega=1$ where they coincide to recover the Taylor-like expansion. We will call $\boldsymbol{G}$ the renormalized kernel matrix. Provided that the spectral radius of $\boldsymbol{G}$ is smaller that one, we can expand $(\boldsymbol{I}-\boldsymbol{K})^{-1}$ into a Taylor series. The first terms are given by

$$
\left\{\begin{array}{l}
\boldsymbol{G}^{0}=\boldsymbol{I} \\
\boldsymbol{G}^{1}=(1-\omega) \boldsymbol{I}+\omega \boldsymbol{K} \\
\boldsymbol{G}^{2}=(1-\omega)^{2} \boldsymbol{I}+2 \omega(1-\omega) \boldsymbol{K}+\omega^{2} \boldsymbol{K}^{2} \\
\boldsymbol{G}^{3}=(1-\omega)^{3} \boldsymbol{I}+3 \omega(1-\omega)^{2} \boldsymbol{K}+3 \omega^{2}(1-\omega) \boldsymbol{K}^{2}+\omega^{3} \boldsymbol{K}^{3} \\
\vdots
\end{array}\right.
$$

The renormalized expansion of $(\boldsymbol{I}-\boldsymbol{K})^{-1}$ is then simply obtained by adding these quantities and multiplying the result by $\omega$ according to Eq. (35). This new expansion is clearly different from the Taylor expansion.

At this stage, it is interesting for our purpose to further generalize our renormalization scheme. Instead of defin- 
ing a single matrix $\boldsymbol{G}$, we can define a whole family of matrices $\left(\boldsymbol{G}_{n}\right)$ by the recursive scheme

$$
\left\{\begin{array}{l}
\boldsymbol{G}_{0}=\boldsymbol{K} \\
\boldsymbol{G}_{1}=(1-\omega) \boldsymbol{I}+\omega \boldsymbol{G}_{0} \\
\boldsymbol{G}_{2}=(1-\omega) \boldsymbol{I}+\omega \boldsymbol{G}_{1} \\
\boldsymbol{G}_{3}=(1-\omega) \boldsymbol{I}+\omega \boldsymbol{G}_{2} \\
\vdots
\end{array}\right.
$$

We deduce then that

$$
\begin{aligned}
(\boldsymbol{I}-\boldsymbol{K})^{-1} & =\omega\left(\boldsymbol{I}-\boldsymbol{G}_{1}\right)^{-1}=\omega^{2}\left(\boldsymbol{I}-\boldsymbol{G}_{2}\right)^{-1} \\
& =\omega^{3}\left(\boldsymbol{I}-\boldsymbol{G}_{3}\right)^{-1}=\cdots
\end{aligned}
$$

so that for any integer $n \in[0,+\infty[$, we have

$$
(\boldsymbol{I}-\boldsymbol{K})^{-1}=\omega^{n}\left(\boldsymbol{I}-\boldsymbol{G}_{n}\right)^{-1} .
$$

Indeed, according to (37), $\boldsymbol{G}_{n}$ is defined by

$$
\boldsymbol{G}_{n}=(1-\omega) \boldsymbol{I}+\omega \boldsymbol{G}_{n-1} .
$$

If we iterate this equation, we obtain finally

$$
\left\{\begin{aligned}
\boldsymbol{G}_{n} & =\left(1-\omega^{n}\right) \boldsymbol{I}+\omega^{n} \boldsymbol{K} \\
& =\boldsymbol{I}-\omega^{n}(\boldsymbol{I}-\boldsymbol{K})
\end{aligned}\right.
$$

We can now add to this family of matrices the families composed of the average and product matrices:

$$
\left\{\begin{array}{l}
\boldsymbol{\Sigma}_{n}=\frac{1}{n+1} \sum_{k=0}^{n} \boldsymbol{G}_{k} \\
\boldsymbol{\Pi}_{n}=\prod_{k=0}^{n} \boldsymbol{G}_{k}
\end{array}\right.
$$

Without going into the details of the derivation that will be part of a forthcoming paper, we find that with the three families of matrices $\left\{\boldsymbol{G}_{n}\right\},\left\{\boldsymbol{\Sigma}_{n}\right\}$ and $\left\{\boldsymbol{\Pi}_{n}\right\}$, we have the renormalization expansions

$$
\begin{aligned}
& -(\boldsymbol{I}-\boldsymbol{K})^{-1}=\omega^{n}\left(\boldsymbol{I}-\boldsymbol{G}_{n}\right)^{-1} \\
& \text { - }(\boldsymbol{I}-\boldsymbol{K})^{-1}=\alpha_{n}\left(\boldsymbol{I}-\boldsymbol{\Sigma}_{n}\right)^{-1} \quad \text { with } \quad \alpha_{n}=\frac{\left(1-\omega^{n+1}\right)}{(n+1)(1-\omega)} \\
& \text { - }(\boldsymbol{I}-\boldsymbol{K})^{-1}=\left(\boldsymbol{I}-\boldsymbol{\Pi}_{n}\right)^{-1} \boldsymbol{\sigma}_{n} \quad \text { with } \quad \boldsymbol{\sigma}_{n}=\omega^{n} \boldsymbol{I}+\boldsymbol{\sigma}_{n-1} \boldsymbol{G}_{n}
\end{aligned}
$$

Interestingly enough, all these renormalized expansions can be related directly to the Neumann expansion we derived in Eq. (18); they correspond respectively to:

$$
\begin{aligned}
& \text { - } \boldsymbol{M}=\boldsymbol{G}_{n} \text { and } \boldsymbol{N}=\omega^{n} \boldsymbol{I} \\
& \text { - } \boldsymbol{M}=\boldsymbol{\Sigma}_{n} \text { and } \boldsymbol{N}=\alpha^{n} \boldsymbol{I} \\
& \text { - } \boldsymbol{M}=\boldsymbol{\Pi}_{n} \text { and } \boldsymbol{N}=\boldsymbol{\sigma}_{n}
\end{aligned}
$$

\section{DISCUSSION}

We have presented in Sec. IV an overview of different finite and infinite multiple expansions that seem good candidates to replace the Watson expansion whose convergence problems have been discussed in Sec. III. Then, in Sec. V we have proposed alternative approaches based on the concept of renormalized expansions. Now, we discuss the pros and cons of each method.

Finite methods have the advantage of being independent of convergence considerations. They are mathematical expansions whose last term will always give the exact result. In contrast to Watson expansion, they are not based on perturbation theory and therefore can be used in any energy range. Correlation expansion has been demonstrated to be more accurate than Watson expansion at the same order [15]. It is implemented in the MsSpec package to compute the cross-section of different spectroscopies [20]. At present all intermediate matrices $\boldsymbol{A}^{\mathrm{ij} \ldots}$ occuring in the expansion are computed. However, many of them hardly contribute to the cross-section and we are developing some approximations to speed-up calculations.

To our knowlege, local-field multiple scattering expansion has never been implemented into a computer code. The idea of local field propagators and transition matrices in itself is rather new in the field and could have some interesting applications. However, when we look at the structure of the expansion itself (see Eq. (29)), the amount of calculation necessary to obtain the first order term is already very important, as it needs the computation of all the $T_{\mathrm{i}}\left[V^{\mathrm{i}}\right]$, the effect of scattering by atom $\mathrm{i}$ in the presence of all the other atoms. In principle, this could be computed by iteration, starting from the quantity $T_{\mathrm{i}}$, but we expect it to be a lengthy process. Moreover, roughly speaking, every term seems to necessitate more or less the same amount of computing. Therefore, despite its extreme originality, we believe that except if very efficient iterative methods are found to compute $T_{\mathrm{i}}\left[V^{\mathrm{i}}\right]$, local-field expansion will remain a curiosity. 
Leverrier-Faddeev method has the disadvantage of being an expansion containing $N_{a t}\left(l_{\max }+1\right)^{2}$ terms compared to $N_{a t}$ terms for the two previous methods. But the method involved is a well-documented algorithm extensively used in the literature, especially to compute generalized inverses. Therefore, provided that it converges rather quickly, it could be a robust alternative to Watson multiple scattering expansion. Tests are underway to assess this expansion.

In the case of infinite expansions, Löwdin second order iteration is very appealing. Starting from an order $n$ approximation of the expansion, Löwdin demonstrates that with a few matrix manipulations, it is possible to obtain the order $2 n+1$ approximation. The problem now is to check how these matrix manipulations (see Eq. (20)) compare in terms of computing time with the matrix inversion. As mentioned earlier, full matrix inversion scales like $N^{3}$, the same as straightforward matrix multiplication. At present, the best matrix multiplication algorithms, such as the Coppersmith-Winograd one scale as $\sim N^{2.37}$ which is a significant improvement over the standard methods. However, expression Eq. (20) shows that the evaluation of $\boldsymbol{S}_{n}^{*}$ necessitates three matrix multiplications, which would make it longer that matrix inversion. Nevertheless, this method could be useful when not all values of the scattering path operator matrix are needed. This is typically the case in PED where only the columns corresponding to the absorber are needed. There, only the corresponding columns of $\boldsymbol{S}_{n} \boldsymbol{K} \boldsymbol{S}_{n}$ will need to be evaluated.

Neumann expansion Eq. (18) is very interesting as it generalizes the idea of renormalized expansion we have derived in Sec. V. It leaves a lot of latitude to seek a satisfying $(\boldsymbol{M}, \boldsymbol{N})$ couple. However, because of the matrix multiplication involved, it would be desirable to choose the couple so that this calculation is reduced to its minimum. One way to achieve this is to take matrix $\boldsymbol{N}$ as being diagonal. This is exactly what we obtain in the two renormalization methods based on the $\left\{\boldsymbol{G}_{n}\right\}$ and $\left\{\boldsymbol{\Sigma}_{n}\right\}$ families we have defined in the previous section.

We consider now some particular values of the renormalization parameters $\omega^{n}$ and $\alpha_{n}$ for which we compute the spectral radius of the associated kernel matrix.

Figure 5 shows the calculation of the spectral radius of the renormalized kernel matrix $\boldsymbol{G}$ for different values of the parameter $\omega$. Here, the black line $\omega=1$ corresponds to the Watson kernel matrix $\boldsymbol{G}_{\boldsymbol{o}} \boldsymbol{T}$. We see clearly in this figure that for some real values of $\omega$ smaller than 1 , the renormalized kernel improves on Watson's kernel (red and green dashed lines).

We turn now to Fig. 6 where some spectral radii corresponding to the $\alpha_{1}$ and $\alpha_{3}$ renormalization schemes are plotted in the case $\omega=0.9$. Here, the effect of the renormalization is even more dramatic, especially in the case $\alpha_{3}$ where the spectral radius is strongly decreased at almost every energy. In particular, it allows a strong convergence at a kinetic energy of $10 \mathrm{eV}$ where the Watson multiple scattering expansion dramatically diverges.

These results demonstrate that renormalization expansion can replace most efficiently standard multiple scattering expansions in calculations. Some work is underway to better understand the convergence properties of these new expansion schemes, to identify the optimal values of $\omega$-renormalized series expansion

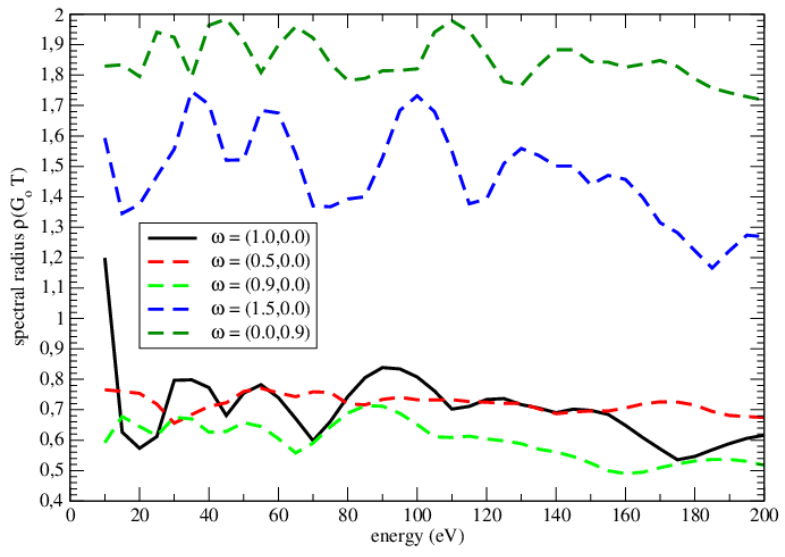

FIG. 5: Values of the spectral radius of the renormalized kernel matrix for different values of the renormalization parameter $\omega$.

$\alpha_{n}$-renormalized series expansion

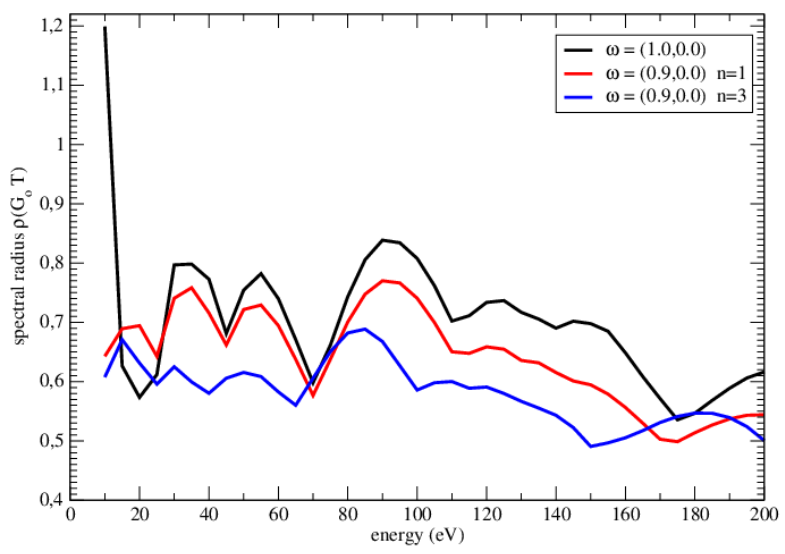

FIG. 6: Values of the spectral radius of the renormalized kernel matrix for different values of the renormalization parameter $\alpha_{n}$.

$\omega$ and to implement these schemes into the MsSpec package [20] to compute the cross-section of spectroscopies.

\section{CONCLUSION}

We have presented a review of possible candidates to replace the Watson multiple scattering expansion. This expansion does not converge at lower energies, and even at higher energies, convergence can be slow in certain cases. As the convergence rate strongly depends on the size of the problem, the need for large clusters to describe nano-objects or nanomaterials does not make this type of expansion a very good candidate for multiple scattering calculations for these objects. We have discussed the pros and cons of different alternative expansions, considering both finite and infinite expansions. For finite expansion, the recently developed correlation expansion seems promising, especially as it can be optimized by making 
use of simple approximations. For infinite expansions, we have proposed a new kind of expansion called renormalization expansion. This type of expansion can be considered as a particular case of the Neumann expansion that was proposed by Löwdin. We have demonstrated in this article the power of this new method that could replace the Watson expansion once its convergence properties are better understood. This will be the object of a forthcoming report.

\section{Acknowledgments}

Part of this work has been funded by European FP7 MSNano network under Grant Agreement $n^{\circ}$ PIRSESGA-2012-317554. Another part was supported by the National Natural Science Foundation of China (project No. 10905067).
[1] D. Sébilleau, R. Gunnella, Z.-Y. Wu, S. Di Matteo, and C. R. Natoli, J. Phys.: Condens. Matter 18, R175 (2006)

[2] J. Korringa, Physica 13, 392 (1947); W. Kohn and N. Rostoker, Phys. Rev. 94, 1111 (1954).

[3] D. Sébilleau, Phys. Rev. B 61, 14167 (2000).

[4] B. L. Györffy, in Fondamenti di Fisica dello Stato Solido (Scuola Nazionale di Struttura della Materia, 1971). B. L. Györffy and M. J. Stott, in Band Structure Spectroscopy of Metals and Alloys, Eds. D. J. Fabian and L. M. Watson (Academic Press, London, 1973), p. 385.

[5] L. D. Faddeev, in Mathematical Aspects of the Three-body Problem in the Quantum Scattering Theory (Israel Program for Scientific Translation, Jerusalem, 1965).

[6] K. M. Watson, Phys. Rev. 89, 575 (1953).

[7] C. R. Natoli, P. Krüger, K. Hatada, K. Hayakawa, D. Sébilleau, and O. Šipr, J. Phys.: Condens. Matter 24, 365501 (2012).

[8] C. R. Natoli and M. Benfatto, J. Phys. (France) C 8, 11 (1986).

[9] D. Sébilleau and C. R. Natoli, J. Phys.: Conf. Series 192, 012002 (2009).

[10] P.-O. Löwdin, J. Math. Phys. 3, 969 (1962).
[11] K. Tanabe, Linear Algebra Appl. 10, 163 (1975).

[12] Y. -L. Chen and X. Chen, Linear Algebra Appl. 308, 85 (2000); J. -J. Climent, N. Thome, and Y. Wei, Linear Algebra Appl. 332-334, 533 (2001).

[13] D. J. Ernst, J. T. Londergan, G. A. Miller, and R. M. Thaler, Phys. Rev. C 16, 537 (1977).

[14] E. R. Siciliano and R. M. Thaler, Phys. Rev. C 16, 1322 (1977).

[15] H.-F. Zhao, D. Sébilleau, and Z.-Y. Wu, J. Phys.: Condens. Matter 20, 275241 (2008).

[16] S. J. Wallace, V. B. Mandelzweig, D. M. Schneider, and J. W. Van Orden, Phys. Rev. C 24, 799 (1981).

[17] D. K. Faddeev and V. N. Faddeeva, Computational Methods of Linear Algebra (Freeman, San Francisco, 1963).

[18] S. -H. Hou, SIAM Rev. 40, 706 (1998).

[19] K. B. Janiszowski, Int. J. Appl. Math. Comput. Sci. 13, 199 (2003).

[20] D. Sébilleau, C. Natoli, G. M. Gavaza, H. Zhao, F. Da Pieve, and K. Hatada, Comp. Phys. Comm. 182, 2567 (2011); http://www.ipr.univ-rennes1.fr/EN/MsSpec 